Padova

\title{
DUE ETIMOLOGIE VENETE ED ISTRIANE
}

\section{VENETO $B(R) U S I C I O$}

Un isolato dialetto del Medio Agordino (La Valle, BL) attesta per 'ginepro' (Juniperus communis L., d'altronde compattamente designato come dzené(i)ver, denéore) il termine bozičo che richiama il busicio dato già per l'area bellunese dal Soravia $(1877,108)$. Il Pellegrini $(1964,28$ e nota 51) ne riscontra il perfetto corrispondente del Veneto orientale buzičco documentato al p. 356 (S. Stino di Livenza) dall'AIS 599 e l'accosta senz'altro al padovano (in realtà piuttosto veneziano di terraferma) brusichio (ch = [č]!) segnalato dal Patriarchi $(1821$, ma 1^ed. 1775) e che si giustificherebbe con l'intrusione di 'bruciare' dal noto uso di bruciare le bacche della pianta per suffumigi, cfr. il ted. regionale Feuerbaum (su cui Marzell 2/1972, 1091) e anche il tipo brusin dell'alta Val di Sole (Pedrotti-Bertoldi 1931, 206s.). Si osservi che anche il grande repertorio poliglotta del Nemnich $(3 / 1794,267)$ dà esplicitamente brusichio per Venezia. Sul tipo e sull'etimo il Pellegrini ritorna più avanti $(1982,185)$ riassumendo la bibliografia precedente e aggiungendo documentazioni dall'ASLEF: $b o$ / $i c ̌$ al p. 172 (Chions, PN) e ancora nel friulano occidentale (in pratica sempre nella zona di confine col veneto) sbrodicio, sbradicio a Budoia (AppiSanson 1970,28); e, quando al problema etimologico, rinviando ancora al solandro brusin - che rientra propriamente nella terminologia dell' 'erica' - e alle osservazioni in proposito di Hubschmid $(1968,329-33)$ ma tenendo ferma la conclusione che *brus jāre 'bruciare' sia soltanto una sovrapposizione paretimologica su una base preromana mal identificabile (*bos -, *bosiclu-), sovrapposizione generata dalle note credenze ed usanze legate al ginepro (su cui cfr. anche Rolland 11/1967, 241 e Marzell 2/1972, 1080, 1086: Reckholder, Rökkraut ecc.). Su fatti del genere ho richiamato l'attenzione io pure nella nota sul lat. juniperus (1987-88, 75s. e note 5,6), a partire dalla chiosa isidoriana (Orig. 17, 7, 35: iuniperus Graece (ossia col rinvio a pŷr) dicta, sive quod ab amplo in angustum finit, ut ignis, sive quod conceptum diu teneat ignem) e soprattutto per quanto concerne i collegamenti magicosacrali che confermano l'attitudine dei suffumigi fatti col legno del ginepro ad allontanare le streghe e i cattivi spiriti e la rinomanza di questa pianta come aromatica, vermifuga, drastica e succedanea di legni più nobili come I'incenso (in particolare per quanto riguarda la specie $J$. sabina $L$.). Da questo punto di vista non ci sono dunque difficoltà ad intuire una sovrapposizione culturale di 'bruciare' all'interno di un tipo che di per sé resta oscuro: d'altra parte una formazione da *bru s jāre con il suffisso -íc(u)lu (unico antecendente possibile di ven. $-i c ̌ c$ : Rohlfs $1969, \S$ 1044) parrebbe strana anche per ragioni linguistiche interne poiché in questi casi la base di derivazione è piuttosto un nome (cfr. it. cavicchio, lenticchia). C'è da osser- 
vare inoltre che di fronte ad un'unica variante con /d/ le altre forme mostrano $/ \mathrm{z} /$ sibilante sonora, fatto significativo per l'area veneta marginale e per l'amfizona veneto-friulana interessate dal tipo che sono caratterizzate dalla presenza delle interdentali $/ \Theta$, $₫ /$ e non di rado quindi dagli scambi tra le continue del tipo $/ z-đ$ (d)/ e simili (nel caso presente facilitati dall'intrusione motivatrice del ven. brusàr). Con ciò si potrebbe ipotizzare dunque una base etimologica che non contenga una continua $/ \mathrm{s}, \mathrm{z} / \mathrm{ma}$ piuttosto $/ \mathrm{d} / \mathrm{e}$ in tal caso non sarebbe difficile indiziare un tipo affine al friul. bedèc m. 'stecco, fuscello', ant. bedeca. f. (Udine, sec. XIV; bedechia, 1425), bedècie f. 'rami inutili della vite che vengono potati' che rinvierei senz'altro (nonostante $\mathrm{i}$ dubbi del Pellegrini in DESF 1, 196) allo slov. bod-, cfr. bosti 'pungere'. Il significato di 'stecco, fuscello, brocco' si conviene infatti al ginepro che è cosi connotato nelle fonti antiche e recenti (cfr. il bell. rustega cioè 'pungente, spinosa'; ted. Dexenstaude, Stechbaum, Nemmnich 3/1794, 267; Merzell 2/1972, 1092). Soccorrono qui in ogni caso vari raffronti sloveni (dal Pleteršnik 1, 38): bodec 'ago' e 'arrestabue (Ononis spinosa L.)', bodek 'ago (di conifere)', 'spazzola', bodčec 'paliuro', bodčica 'letto di aghi di conifere', bode' 'arrestabue' ma soprattutto con vocalismo / $\mathrm{i} /$ bodic 'Murex tenuispina', bodica 'ago, aculeo', bodic 'ago (di conifere)', 'carlina (Carlina acaulis L.)' e 'Xanthium spinosum', bodičje 'id.' e 'agrifoglio (Ilex aquifolium L.)', bodika 'id.', tutte piante evidentemente spinose o ruvide. Si aggiunga qui anche il cr. bodelj 'sorta di cardo (Dipsacus silvestris L.)', bodilica 'cirsio', bocka 'Xanthium', bodalj 'cardo', cfr. bodlja 'spina'; e con vocalismo /a/ bad-, badalj 'spina', badelj 'cardo'; pol bodlak 'cardo' ecc. (ERHSJ 1, 191s.). Dunque bodič, bodic sembrano un'ottima base etimologica del veneto marginale busicio e varianti anche se non sono del tutto chiare le trafile che dallo slavo hanno portato questa base in una sede relativamente lontana.

\section{ISTRIANO PIMPIGNO}

Nel suo profilo storico-etimologico della latinità istriana la Cerasuolo Pertusi (in stampa) include tra le voci istriane prevenete pimpigno 'la punta in pietra sopra il secondo coperto (quello conico) della casita', pimpignól 'la pietra che tiene unito il tetto delle casite' (confermato dal Rosamani), pimpilín 'la pietra finale della casita', voci isolate la cui spiegazione partirebbe da un campanil (o campa(g)nòl) con dissimilazione (?) regressiva a distanza $/ \mathrm{k}-\mathrm{p} />/ \mathrm{p}-\mathrm{p} /$ e successiva metatesi per quanto riguarda pimpilín $(<*$ pimpiníl): il semantismo avrebbe un facile riscontro nel triestino ant. trullo 'campanile' (propriamente 'costruzione conica'). Si tratta di un termine che deve la sua conservazione ad una particolarità architettonica propria delle costruzioni rustiche istriane ma non certo privo di raffronti nel resto della Romània: il REW 6422 *perpannium 'pietra a piombo' dà infatti fr.ant. pierre perpaigne, fr. parpaing (da cui sic. parpañu e sp. perpiaño), surselv. parpaun, engad. partaun; il Faré aggiunge sic. purpañu, parpañu 'regolo' e cita dal Parlangèli il salent. purpitagnu 'muro sottile tra due vani, grossa pietra squadrata da costruzione', che vorrebbe un *perpedāneu (cfr. pedāneus, REW 6343); il terzo volume del VS conferma 
ora ampiamente pappagnu, parpagnu 'regolo', a-pparpagnu 'a piombo', 'perfettamente parallelo (di muro)' ecc. (comunque un normannismo). Molto materiale in più si ottiene dal FEW 8, 258s. che propone sotto un ricostruito *perpetāneus 'ininterrotto' il fr. ant. (1291) perpein 'senso di lunghezza (di una pietra da costruzione)', fr. parpain (1339) 'pietra messa in taglio' e numerosi riscontri dialettali; parpeing (1606) 'pietra di sostegno', 'fila di pietre'; pierre de perpagne (1460), parpaigne (1578) ecc., con derivati del tipo fr. med. parpignel, parpignot (Fiandre, 1456, 1510) 'pietra posta dietro il focolare' ecc.: secondo il FEW questa famiglia si lega senza dubbio a quella dello sp. perpiaño 'pietra passante (in un muro)' e dell'italiano merid. (salentino) perpitagnu 'parapetto di pietra del balcone', 'pietra squadrata da costruzione', Otranto perpetagno, Taranto purpitagno 'muro sottile tra due vani' e più in là dell'alto engad. partaun 'architrave', Bergün parpaun, surselv. id. 'barriera' e gli etimi proposti in un primo tempo ('perpannium da pannus; *perpedāneus) dovrebbero cedere all'intuizione di Alessio ( $R L i R ~ 17,192)$ che parte dal lat. perpes, ëtis 'ininterrotto, collegato' donde appunto '*perpetāneus. Il Corominas (DCELC 3, 748-50; 4, 1062s.) sottolinea la specificità srchitettonica dello sp. perpiaño (primariamente aggettivo associato ad arco: 1506, Palencia; 1552, Villalpando), forse forma dialettale aragonese o leonese col trattamento locale di /ĕ/ (il portoghese perpianho sarebbe a sua volta castiglianismo o leonesismo), riproponendo dubitativamente un etimo lat. tardo perpěndium 'equilibrio, posizione a piombo' (cfr. perpendicŭlum 'filo a piombo') a suo tempo già proposto con vari argomenti da G. Paris: da scartare sarebbe invece, per carenza di fondamento semantico, la proposta *perpedāneu di Tuttle (RRQ 7, 351) e Malkiel (AGI 36, 6872) che pure si adatterebbe bene soprattutto agli esempi salentini (dove /t/ intervocalico è facilmente prodotto di strengthening fonologico ossia di una regola antilenitiva: cfr. nitu, pete ecc.). Le corrispondenze catalane perpany (Maiorca), perpeny (Minorca), ant. (1435, Valencia) perpeany parlerebbero comunque in favore di quest'ultimo: e infatti il Corominas (DECLIC 6, 508) conferma l'ipotesi classificando sotto peu piede' queste voci nel senso primitivo di 'pietra, elemento strutturale che va da un lato all'altro'. Le forme istriane, che entrano di diritto in questa costellazione, non si giustificano direttamente da quest'etimo ma richiederebbero semmai una retroformazione di pimpigno dal derivato pimpignól (/i/ tonico della forma semplice non è infatti spiegabile altrimenti): e in ogni caso, sia per fonetica che per semantica, troverebbero la migliore collocazione esattamente sotto perpëndium o ancor meglio sotto ${ }^{*}$ per pen di ār e, come esiti deverbali (che ad eccezione della variante valenciana darebbero ragione dell'intera famiglia romanza).

Nota: sigle ed abbreviazioni bibliografiche sono quelle generalmente in uso. Per il resto cfr. AppiSanson 1970 = E. e R. Appi - U. Sanson, Aggiunte al 'Nuovo Pirona'. Vocabolario friulano - Zona di Budoia. Udine, 1970; Cerasuolo Pertusi = M.R. Cerasuolo Pertusi, Il contributo dell'etimologia per la storia della neolatinità istriana, in corso di stampa; Hubschmid $1968=\mathrm{J}$. Hubschmid, Bezeichnungen für Erika und andere Sträucher, Gestrüpp und Auswüchse, Vox Romanica 27/2, 319-59; Marzell = H. Marzell, Wörterbuch der deutschen Pflanzennamen, Leipzig 1942ss.; Nemnich = P.A. Nemnich, Allgemeines Polyglotten - Lexikon der Naturgeschichte, Hamburg 1793ss.; Patriarchi 1821 = G. Patriarchi, Vocabolario veneziano e padovano, co' termini e modi corrispondenti toscani, Padova; Pedrotti-Bertoldi $1931=\mathrm{G}$. Pedrotti e V. Bertoldi, 
Nomi dialettali delle piante indigene del Trentino e della Ladinia Dolomitica, Trento; Pellegrini 1964 = G.B. Pellegrini, Introduzione a G.B. Rossi, Flora popolare agordina, Firenze; Pellegrini 1982 = G.B. Pellegrini e A. Zamboni, Flora popolare friulana, Udine; Rohlfs $1969=$ G. Rohlfs, Grammatica storica della lingua italiana e dei suoi dialetti. III, Sintassi e formazione delle parole, Torino; Rolland = E. Rolland, Flore populaire de la France, Paris 1877-1911; Soravia $1877=\mathrm{P}$. Soravia, Tecnologia botanico-forestale della provincia di Belluno, Belluno; VS = Vocabolario siciliano, promosso da G. Piccitto. Catania-Palermo, 1977ss.; Zamboni 1987-88 = A. Zamboni, Lat. iuniperus: note specifiche e riflessioni generali sul contatto latino-germanico. Incontri linguistici $12,73-89$.

Povzetek

\section{BENEŠKA IN ISTRSKA ETIMOLOGIJA}

Ǐče se etimologija za ben. $b(r) u s i c i o$ (Juniperus communis); ker se beseda v podobni glasovni podobi pojavlja tudi v vzhodni Benečiji, se avtor nagiba $\mathrm{k}$ mnenju, da je morda vendar treba upoštevati slovensko podstavo bod-. Pri tem navaja številne primere iz Pleteršnika: bodec, bodek, bodež, bodič. Istrsko predbeneško pimpigno dokazuje arhitekturno svojskost istrske hiše. Verjetni vir je lat. perpetāneus (perpes 'nepretrgan'), cf. lat. perpèndium in *perpendiðre. 